\title{
Improving 3D reconstructions of macromolecular conformations
}

\author{
M. Kazemi ${ }^{1}$, C. O. S. Sorzano ${ }^{1}$, A. Des Georges ${ }^{2}$, J. M. Carazo ${ }^{1}$, J. Vargas ${ }^{3}$ \\ ${ }^{1}$ Biocomputing Unit, Centro Nacional de Biotecnología-CSIC, \\ C/ Darwin 3, 28049, Cantoblanco (Madrid), Spain \\ ${ }^{2}$ Biochemistry and Molecular Biophysics, Columbia University, \\ New York, NY 10032, USA \\ ${ }^{3}$ Departament of Anatomy and Cell Biology, McGill University \\ 3640 Rue University, Montréal, QC H3A 0C7, Canada \\ *Correspondence to javier.vargasbalbuena@mcgill.ca
}

Cryo-electron microscopy using single particle analysis requires the computational averaging of thousands of projection images captured from identical macromolecules. However, macromolecules usually present some degree of heterogeneity showing different conformations. Reconstruction of these conformations is essential to infer the macromolecular function and understand how it works. Computational approaches are then required to classify heterogeneous single particle images into homogeneous sets at different conformations. Nonetheless, sometimes the attainable resolution of reconstructions obtained from these smaller homogeneous sets is compromised because of reduced number of particles or lack of images at certain macromolecular orientations. In these situations, the unique solution to improve map resolution is returning to the electron microscope and collect more data. In this work, we present an approach to overcome this limitation for heterogeneous datasets. Our method is based on moving particles between different conformations using an optical flow approach. Particles are then merged in a unique conformation obtaining reconstructions with improved resolution, contrast and signal-to-noise ratio. 